\title{
Intravenous contrast medium extravasation: systematic review and updated ESUR Contrast Media Safety Committee Guidelines
}

\author{
Giles Roditi ${ }^{1}$. Nadir Khan ${ }^{1}$ - Aart J. van der Molen ${ }^{2} \cdot$ Marie-France Bellin $^{3} \cdot$ Michele Bertolotto $^{4} \cdot$ Torkel Brismar $^{5}$. \\ Jean-Michel Correas ${ }^{6} \cdot$ llona A. Dekkers $^{2} \cdot$ Remy W. F. Geenen $^{7}$. Gertraud Heinz-Peer ${ }^{8}$. Andreas H. Mahnken ${ }^{9}$. \\ Carlo C. Quattrocchi ${ }^{10} \cdot$ Alexander Radbruch $^{11}$. Peter Reimer ${ }^{12}$. Laura Romanini ${ }^{13}$. Fulvio Stacul ${ }^{14}$. \\ Henrik S. Thomsen ${ }^{15}$. Olivier Clément ${ }^{16}$
}

Received: 28 May 2021 / Revised: 29 August 2021 / Accepted: 14 October 2021 / Published online: 17 February 2022

(c) The Author(s) 2022

\begin{abstract} Key Points

- Risk of extravasation is dependent on scanning technique and patient risk factors.

- Diagnosis is mostly clinical, and outcomes are mostly favourable.

- Referral to surgery should be based on clinical severity rather than extravasated volume.
\end{abstract}

Need for a review Guidelines for management and prevention of contrast media extravasation have not been updated recently. In view of emerging research and changing working practices, this review aims to inform update on the current guidelines. Areas covered In this paper, we review the literature pertaining to the pathophysiology, diagnosis, risk factors and treatments of contrast media extravasation. A suggested protocol and guidelines are recommended based upon the available literature.

Keywords Contrast media $\cdot$ Extravasation of diagnostic and therapeutic materials $\cdot$ Risk factors $\cdot$ Prevention and treatments

*OCEBM Levels is a critical appraisal tool designed by an international group which allows evaluation of the strength of evidence for a range of clinical questions, i.e. prevalence, accuracy of diagnostic tests, prognosis, therapeutic effects, rare and common harms, and usefulness of screening.

Giles Roditi and Nadir Khan Both contributed equally to this work

Olivier Clément

olivier.clement@aphp.fr

1 Department of Radiology, Glasgow Royal Infirmary, Glasgow, UK

2 Department of Radiology, Leiden University Medical Center, Leiden, The Netherlands

3 University Paris-Saclay, AP-HP, University Hospital Bicêtre, Service de Radiologie, BioMaps, Le Kremlin-Bicêtre, France

4 Department of Radiology, University Hospital Trieste, Trieste, Italy

5 Department of Clinical Science, Intervention and Technology, Unit of Radiology, Karolinska Institutet and Department of Radiology, Karolinska University Hospital in Huddinge, Stockholm, Sweden

6 Université de Paris, AP-HP, Groupe Hospitalier Necker, DMU Imagina, Service de Radiologie, Paris, France

7 Department of Radiology, Northwest Clinics, Alkmaar, The Netherlands
8 Department of Radiology, Landesklinikum St. Pölten, St. Pölten, Austria

9 Department of Diagnostic and Interventional Radiology, Marburg University Hospital, Marburg, Germany

10 Imaging Center, Unit of Diagnostic Imaging and Interventional Radiology, Università Campus Bio-Medico Di Roma, Rome, Italy

11 Department of Radiology, Clinic for Diagnostic and Interventional Neuroradiology, Bonn, Germany

12 Department of Radiology, Institute for Diagnostic and Interventional Radiology, Klinikum Karlsruhe, Karlsruhe, Germany

13 Department of Radiology, ASST Cremona, Cremona, Italy

14 Department of Radiology, Ospedale Maggiore, Trieste, Italy

15 Department of Radiology, Copenhagen University Hospital Herlev, Copenhagen, Denmark

16 Université de Paris, AP-HP, Hôpital Européen Georges Pompidou, DMU Imagina, Service de Radiologie, 20 Rue LeBlanc, 75015 Paris, France 


\section{Abbreviations}

$\begin{array}{ll}\text { CM } & \text { Contrast media } \\ \text { CMEX } & \text { Contrast media extravasation } \\ \text { CT } & \text { Computed tomography } \\ \text { EDA } & \text { Extravasation detection accessory } \\ \text { GBCA } & \text { Gadolinium-based contrast agent } \\ \text { IBCM } & \text { Iodine-based contrast media } \\ \text { IV } & \text { Intravenous } \\ \text { MeSH } & \text { Medical subject headings } \\ \text { MRI } & \text { Magnetic resonance imaging } \\ \text { OCEBM } & \text { Oxford Centre for Evidence-Based Medicine* }\end{array}$

\section{Introduction}

Contrast media extravasation (CMEX) is a complication where there is leakage of intravenously administered contrast agents (either iodine or gadolinium-based), into the surrounding soft-tissues [1]. This can vary in severity from minor discomfort to compartment syndrome, skin ulceration and necrosis. A recent observational study of 142,651 participants undergoing CT scans showed a CMEX incidence of $0.23 \%$ [2] whilst a systematic review by Behzadi et al. accounting for 17 studies in 1,104,872 patients found CMEX rates of $0.2 \%$ [3]. Rates of serious CMEX seem much lower, only described in case reports and case series [4-7] although this probably reflects under-reporting.

CMEXs are thought to be one of the most frequent adverse events in radiology but are much less studied than others such as contrast-associated acute kidney injury [8-10]. Whilst CMEX does not usually lead to significant morbidity, the rare, serious complications of compartment syndrome, skin ulceration and tissue necrosis are important to recognise [4-6]. Furthermore, even what may be clinically regarded as minor CMEX will be perceived as important by the patient and contribute to feelings of dissatisfaction at a stressful time since "something has gone wrong" with their care.

Previous guidelines around CMEX from the Contrast Media Safety Committee (CMSC) of the European Society of Urogenital Radiology (ESUR) published in 2002 related to older contrast injection protocols [1]. Since then, several prospective studies investigating CMEX risk factors and management as well as systematic reviews have been published [2, 3, 11-13]. In this paper, we aim to inform update of the CMSC guidelines by performing a systematic review and provide recommendations.
Fig. 1 Search and selection procedures

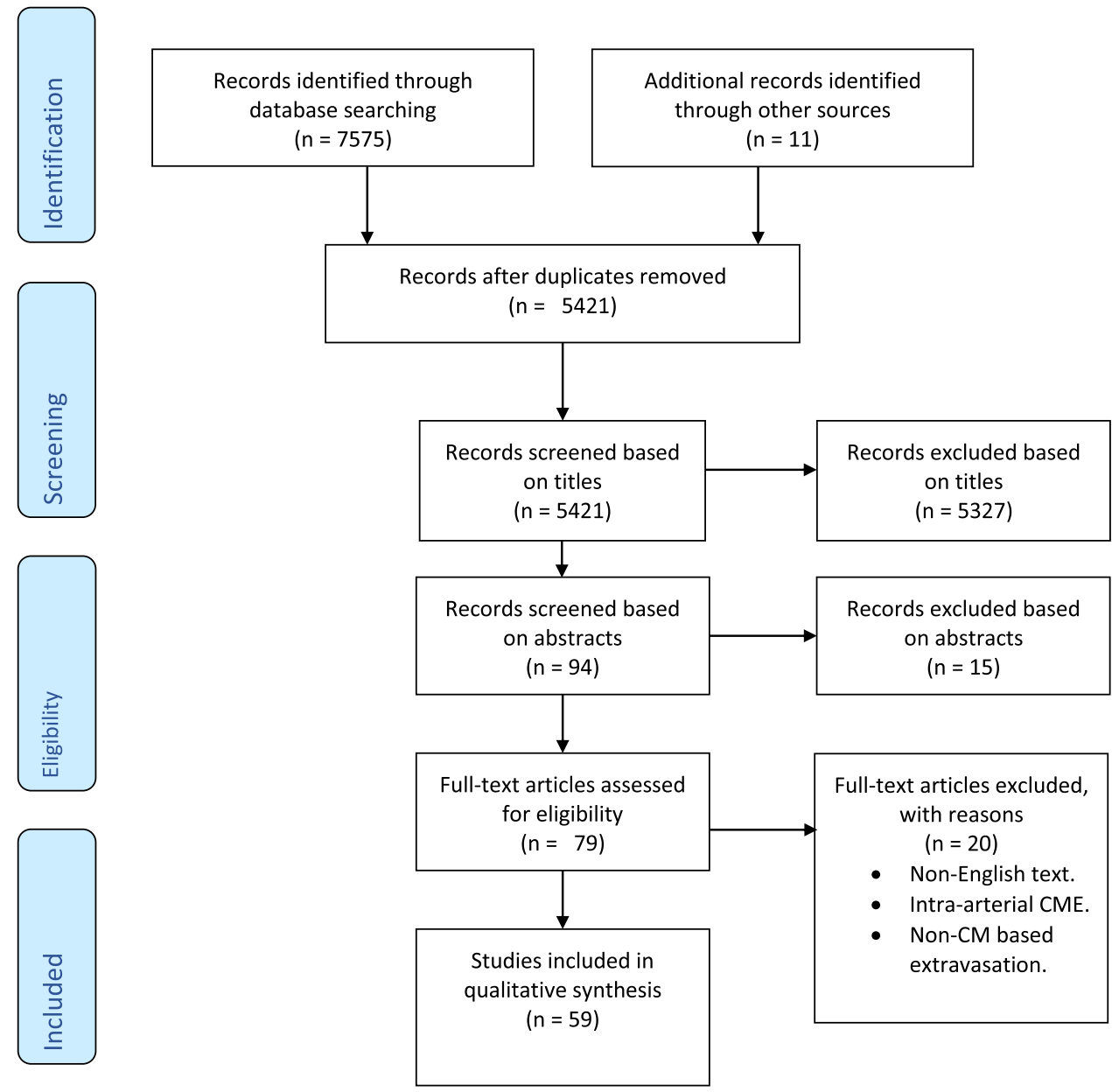




\section{Methodology}

Authors prepared seven clinical questions in Patient-Intervention-Comparison-Outcome (PICO) format [14]. A systematic literature search of PubMed and Scopus from 1 January 1990 through to 6 February 2021 with MeSH terms as described in the search strategy (Appendix 1) was performed by a radiology trainee (N.K.) with previous meta-analysis experience and a radiology consultant (G.R.). Abstracts were reviewed for relevance, references scrutinised and crossreferenced. Only articles in English pertaining to intravenous CMEX in context of CT and MRI were considered. References from previous CMSC guidelines were included. CMEXs associated with contrast-enhanced ultrasound were excluded as no cases have been reported in the literature [15-17]. Studies assessing intra-arterial CMEX and noncontrast media-based extravasation were also excluded (Fig. 1 flow-chart of article selection and final number of articles).

Risk of bias of each study included was graded according to National Institutes of Health (NIH) study quality assessment tools. The strength of recommendation of different risk factors, diagnosis, detection and management of CMEX were graded according to OCEBM Levels of Evidence Working Group "The Oxford 2011 Level of Evidence" (Appendices 2 and 3). Characteristics, risk of bias and levels of evidence for each included study are outlined in Appendix 4.

Studies were synthesised and summarised narratively.

\section{What are the pathophysiologic mechanisms of CMEX?}

Three main processes underpin the mechanisms of CMEX [18]. The first model describes fluid escaping into perivascular tissue through extraluminal dislocation or break of the catheter tip. The second model describes a leak through the puncture site of a correctly placed cannula leading to extravasation. The third model envisages shear stresses and total pressure from the jet at the vessel wall directly leading to vessel disruption and extravasation [19].

The degree to which tissue damage occurs due to CMEX is dependent on the CM used (osmolality, cytotoxicity and extravasated volume) plus location of the cannula; more damage occurs with involvement of tight sub-fascial compartments compared to looser subcutaneous layers [20]. An acute inflammatory response which peaks up to $48 \mathrm{~h}$ after injection can be followed by several weeks of chronic inflammation [21]. However, the majority resolve in 2-4 days with resorption of CM extravasate thought to be primarily by the lymphatic system [13].
Consequently, much research has been based on preventing cannula dislodgement and exploring the impact of variables that increase risk of leakage from the puncture site.

\section{How to detect extravasation?}

With CMEX contrast escapes and infiltrates the interstitium during injection. This is mostly a clinical diagnosis and routine use of imaging is not indicated for its detection.

Non-physicians are usually first responders to CMEX, i.e. radiology technicians/radiographers and healthcare support workers. Hence, it is recommended that imaging departments follow a protocol which allows identification of at-risk patients, easy detection of CMEX, awareness of monitoring needs and effective management of extravasation by a wide range of staff [22]. As an excerpt from multiple publications, the following algorithm is proposed (Table 1). Outpatients with extravasation should be kept under observation in the department until a physician is satisfied that signs and symptoms are resolving such that intervention will not be needed and the patient allowed home.

We summarise three main ways of detecting CMEX in Table 2 .

Many vendors have developed Extravasation Detection Accessories (EDA) which are sensors that allow detection and interruption of automated injection in the event of an extravasation. Dykes et al. demonstrated the use of EDA resulted in smaller volumes of CMEX when they occurred compared to when no EDA was utilised (1,085 CMEX events in 454,497 CT exams across 58 radiology practices) [23]. However, incidence of CMEX did not change and importantly some large volume extravasations still occurred with 28 patients suffering 50-99 $\mathrm{ml}$ of CMEX and 4 patients experiencing $>100 \mathrm{ml}$ of extravasate (unlike studies by Powell et al. and Birnbaum et al.). However, with multiple centres, it was difficult to compare the performance of different EDAs against each other since the centres used different EDAs (types not specified) [23].

One quality improvement project and three case-series have investigated EDA use; each assessed a different device [24-27]. Two studies only published preliminary results and did not specify sensitivity or specificity [28, 29]. These devices rather than fully preventing extravasation are designed to detect it early and so prevent a mild extravasation from becoming moderate or even severe. A summary of the clinically tested EDA devices is provided in Appendix 5.

\section{Is documentation of extravasation needed?}

Proper documentation of CMEX is crucial [12]; at the patient level, this allows early recognition of deterioration 
and aids interpretation across a broad range of healthcare staff. More broadly, documentation at an institutional level allows auditing which can improve local working practices. Dykes et al. showed that the rates of observed CMEX reduced as a result of multi-institution audit; however, they did not impact the volume of extravasate when extravasation did occur [23].

With severe extravasations, imaging documentation may be helpful [30]. Plain radiographs with 2 orthogonal views, 2-plane CT topogram or indeed cross-sectional images with the CT or MRI scanner before removing the patient from scanner table are recommended to assess for compartmentalisation (subfascial vs subcutaneous) and extent of extravasate $[21,31]$.

\section{What are the risk factors for extravasation?}

\section{Technique related}

Peripheral IV cannula type, size and location Six studies evaluated the influence of the physical properties of cannulae; none were prospective randomised trials. A pseudorandomised trial compared between a fenestrated $20 \mathrm{G}$ and a non-fenestrated $18 \mathrm{G}$ cannula which showed no effect on CMEX rates or volume [32]. Furthermore, retrospective cohort studies have shown that extravasation rates were higher with $22 \mathrm{G}$ compared to $20 \mathrm{G}$ and $18 \mathrm{G}$ cannulas, but no significant difference between 18 and 20G cannulas [3335]. In addition, these studies have reported no difference in extravasate volume between the use of different cannula sizes. In a paediatric prospective cohort study, there was no effect of cannula size on CMEX [36]. Location of the peripheral cannula was an important risk factor in three studies with higher rates of extravasation when placed in a vein in the dorsum of hand compared to an antecubital fossa vein. However, higher volume of extravasate was observed with injections in antecubital fossa versus hand veins despite the higher incidence at the hand/wrist, likely related to use of higher flow rates and delayed recognition of CMEX at the antecubital fossa. Overall, injection sites in the lower limbs and small distal veins are less optimal.

CVCs, PICCs and power injectable ports Central venous catheters (CVC-tunnelled or non-tunnelled), totally implantable vascular access devices, haemodialysis catheters and peripherally inserted central catheters (PICCs) are increasingly used for patients in critical care, on chemotherapy or long-term antibiotics. Of course these patients often require regular cross-sectional imaging with IV contrast. Power injector compatible versions of these have been shown to be safe [37], with a low $1 \%$ reported risk of adverse incident. Each type of catheter has maximal flow rate and pressure 
Table 2 Detection of contrast media extravasation

1. Direct Patient Observation: Technician/radiographer in room or video monitoring as well as self-reporting by the patient. Observation of monitoring scans for expected contrast arrival and completed scans for enhancement.

2. Pressure Monitoring: Power-injectors for contrast media (CM) administration now have pressure monitoring systems with graphic displays and flow profile previews which can automatically or semi-automatically stop the CM injection if increased resistance or faults within the system are encountered. One of the promoted benefits of this technology is CM extravasation prevention/minimisation although no published studies have specifically explored their impact to date.

3. Extravasation detection accessories: Some vendors have developed sensors to detect CM extravasation amongst other capabilities.

limits detailed by the manufacturer. However, there remains variability in practice and persistent concern regarding complications due to potentially high pressures achieved using pump injections of CM and how very few catheters have been studied for use with power injectors [38, 39]. Although rare, extravasation from central catheters can lead to significant morbidity, i.e. mediastinal extravasation, haematoma and cardiac arrhythmias.

Prior to the advent of power-injectable CVCs, the Medical and Healthcare Products Regulatory Agency (MHRA) in the UK published recommendations regarding rates and volumes for CM injections. However, these have been withdrawn and current advice is to follow specific manufacturer guidance. Plumb and Murphy recommended a maximum flow rate of $2.0 \mathrm{ml} / \mathrm{s}$ for CVC and to use the distal lumen if a multi-lumen CVC is in place [37]. A CT topogram is a good method to quickly evaluate catheter tip positioning post $\mathrm{CM}$ administration. Overall, extravasation injury is extremely rare with the main concern pertaining to mispositioning and tube integrity. Power injectable TIVADs have a growing role; a retrospective study did not find any incidence of CMEX in 307 patients studied over 4 years with injection rates of 3-5 ml/s employed. Although the evidence base for the use of these power injectable ports is limited, there has been no CMEX demonstrated [40]. Another retrospective study found similar findings [41] whilst Rigsby et al. also found pressure-limited power injection through central lines in children to be safe (no complications, 63 patients aged 0.3-22 years) [42].

\section{Power injection compared to manual injection via peripher-} ally inserted IV cannula Sinan et al. did not find any significant difference in extravasation rates in patients receiving $\mathrm{CM}$ via power injection vs. manual injection [34]. A study by Barrera et al. of power injectors in children also found low rates of extravasation and long-term injury [43]. Preparation of the injector system is key to minimise the risk and departments should follow a standard protocol, i.e. correct alignment and clearing of syringe and pressure tubing.

Infusion rate and volume of $\mathrm{CM}$ Six studies have assessed rate and volume impact on CMEX. A randomised controlled trial by Kok et al. demonstrated no significant difference in CMEX when assessing different flow rates ( $8.3 \mathrm{ml} / \mathrm{s}, 6.7 \mathrm{ml} / \mathrm{s}$ and $5.4 \mathrm{ml} / \mathrm{s}$ ) [44]. However, different $\mathrm{CM}$ were used to ensure equivalent iodine delivery rate and load remained constant. Furthermore, a major confounding variable was that catheter size was not kept constant. Findings from other research groups [34, 34, 45, 46] are similar with the caveat that all have confounding factors of different cannula sizes and type of CM used. Whilst Wienbeck et al. found a statistically significant increase of extravasate volume with the use of high flow rates, Moreno et al. showed the converse of reduced extravasate volumes with higher flow rates [46]. The use of EDAs in this study appeared to reduce volumes of extravasate at higher rate injections.

Cannula insertion technical factors A non-randomised retrospective study found higher rates of extravasation in patients who had ultrasound-guided cannula insertion $(3.6 \%)$ vs. standard insertion $(0.3 \%)$. However, the numbers of patients in each group were drastically different-364 in the ultrasound-guided cannula inserted group and 896 in the standard cannula inserted group (2\% of a random sample of 40,143 patients). The difference in results in this study is likely due to confounding variables; i.e. prior failure of standard insertion and with deeper veins there is potentially a shorter length of intravascular cannula hence greater potential for dislodgement upon injection [47]. The type of healthcare worker who inserts the IV cannula was investigated by Sinan et al. which showed that CMEX rates were higher when inserted by non-radiology staff $(0.3 \%)$ as compared to radiology staff $(0.2 \%)$. However, this difference did not demonstrate statistical significance and a number of confounders could explain this difference [34]. Another study evaluated extravasation between newly inserted cannula vs. use of an existing cannula for $\mathrm{CM}$ injection with a difference in rates and a reduced volume of extravasate observed with those freshly sited (40.6 $\mathrm{ml}$ vs. $63.1 \mathrm{ml}, p=0.0005)$ [46], despite older cannulae being checked for flush efficacy. Therefore, consideration should be given to placing a new cannula for at-risk patients to reduce potential impact of any extravasation. 
Chemical properties of $\mathrm{CM}$ Three main contrast media properties have been studied in relation to CMEX: osmolarity, charge and viscosity.

Osmolarity-A relationship has been demonstrated between higher osmolarity CM (600, 1500 and $2100 \mathrm{mOsm} /$ $\mathrm{kg} \mathrm{H}_{2} \mathrm{O}$ ) and cellular lysis which is thought to be a factor in the degree of tissue damage caused by extravasation. This has only been studied in animal models where old ionic CM (such as sodium and meglumine ioxithalamate) was used [48, 49]. Human clinical studies with non-ionic CM are lacking.

Ionicity-Charge can influence extravasation in that ionic $\mathrm{CMs}$ are thought to increase complications; however, ionic IBCMs are no longer used for intravenous studies. Studies have shown that non-ionic IBCMs (such as Iopamidol 300 and 370, and iohexol 300 and 350) are well tolerated in humans $[30,49,50]$. On the other hand, most GBCAs are ionic so this can potentially play a role in the severity of extravasation injury although volumes used are much lower and again human clinical studies are lacking.

Viscosity-This is thought to influence the likelihood of extravasation occurring. A computational fluid dynamics study by Sakellariou et al. demonstrated the potential for increased incidence of extravasation with more viscous CM, especially in CT angiography when performed with smaller peripheral cannulas [19]. A viscosity of $>9.4 \mathrm{mPa} \cdot \mathrm{s}$ demonstrated increased risk of extravasation in the study by Hwang et al. [2]. An inverse relationship exists between viscosity and temperature in liquids; hence, warmed CMs are less viscous and offer lower resistance. Davenport et al. evaluated the role of warming $\mathrm{CM}$ to 37 degrees vs. $\mathrm{CM}$ at ambient temperature in $>20,000$ patient contrast injection trialled with two contrast media. There was no significant difference in CMEX incidence between warmed versus ambient temperature Iopamidol 300. However, the higher viscosity Iopamidol 370 had an increased incidence of CMEX that was reduced (becoming similar to that with the lower viscosity $\mathrm{CM}$ ) when the CM was warmed. There was, however, no effect on the volume of extravasate when CMEX occurred [51]. A recent study evaluating safety of Iomeprol 400, when warmed to 37 degrees, reported CME rate of $0.71 \%$ out of a total of 3,514 injections, with site and/or size of cannula not influencing CMEX rates [52]. However, the authors found inability to aspirate blood through the cannula significantly correlated with incidence of CMEX.

Overall, this suggests that using CM with lower viscosity and/or warming $\mathrm{CM}$ and/or diluting/mixing $\mathrm{CM}$ with saline has a preventative role in CMEX.

Gadolinium based contrast agents There is much less data on rates of CMEX for MRI; the incidence of CMEX is reported as approximately $0.06 \%$ with no serious complications described-likely due to low infusion rates and lower CM volumes compared to IBCM uses. Theoretically, the extravasation of GBCAs could lead to oedema, necrosis or haemorrhage, potentially exacerbated due to their ionicity and higher osmolarity when compared to IBCM, although this does not seem to be borne out in practice [3, 48].

\section{Patient-related factors}

Based on a meta-analysis of 356,582 patients by Ding et al., females and older patients ( $>60$ years) are at greater risk of developing CMEX; however, gender and age have no impact on the volume of CM extravasation when it does occur [11]. In-patients are at an increased risk compared to those having outpatient scans, especially intravenous drug users and patients with recent hospitalisation [11]. Further risk factors include those patients with an inability to communicate, fragile veins, compromised venous and/or lymphatic drainage and obesity [1].

It is suggested based on the risk factors discussed in Table 3 that the measures outlined in Table 4 will greatly reduce the chances of extravasation occurring and minimise severity when it occurs.

\section{What are the proposed treatments?}

The untreated sequelae of severe CMEX are as follows: increased intra-compartmental pressure (compartment syndrome), with subsequent risk of ischemia due to venous congestion and low arterial gradient causing disproportionate necrosis, severe neurovascular compromise or even limb loss. Treatments to avoid these serious complications can be divided into passive conservative measures and more active therapies (Table 5).

Studies assessing conservative methodologies to treat extravasation events are few and not of robust quality in terms of applicability for radiology, i.e. laboratory-based studies or investigations of extravasation of cytotoxic drugs rather than contrast media. Most recommendations are based on "good clinical practice". The aim of conservative measures is to reduce the morbidity associated with CMEX. The evidence base for use of invasive treatments is limited with mostly retrospective studies, small case numbers, lack of control groups, data based on older CM or even not CMEX.

It is important that clear instructions to the patient who has suffered CMEX are given regarding when to seek additional medical care if there are worsening symptoms. A patient information leaflet is recommended, available in the languages appropriate for the institution. Patients should be warned about red flag signs and symptoms which are as follows: 
- Increased swelling or pain

- Increased redness

- Change in sensation in the affected limb

- Skin ulceration or blistering

In Table 6, we outline a suggested protocol for the management of contrast media extravasations.

\section{When to get a surgical consult?}

Compartment syndrome is the most serious among the consequences of CMEX. It is extremely rare with less than 12 cases reported in a recent literature review however must be suspected if patient complains of severe pain and/or neurovascular compromise [53]. Signs which would necessitate fasciotomy being painful active flexion, passive extension, neurosensory disturbance and increasing swelling. Locations which are most at risk are volar forearm and the hand. Whilst a radiologist can attend to mild cases, in more severe cases, a surgeon should be urgently consulted, and fasciotomy should be performed within $2 \mathrm{~h}$ to avoid muscle necrosis and nerve damage. Surgery performed within 90 to $300 \mathrm{~min}$ has been reported to conserve all extremities with favourable prognosis [54]. Sbitany et al. in a study of 102 cases of CMEX used a threshold of $30 \mathrm{ml}$ extravasate as a referral prompt [55] and none required surgery, even in the $10 \%$ with $>100 \mathrm{ml}$ CMEX volume. Wang et al. reported similar findings where even extravasate volumes of up to $150 \mathrm{ml}$ of non-ionic IBCM were conservatively managed, suggesting no discrete threshold extravasate volume should be set [56]. Dykes et al. suggest that larger extravasated volumes correlate with moderate-to-severe injuries, but the data does not support using a volume threshold to determine surgical referral or more aggressive management [23]. Nevertheless, in unusual cases with very large volumes of extravasate ( $>150 \mathrm{ml})$, the consensus remains that surgical consult is appropriate.

Overall, the decision to refer for surgical intervention should be a clinical one-i.e. based on red flag signs and symptoms, rather than arbitrary CMEX volumes.

\section{Conclusion}

This review highlights the key up-to-date evidence pertaining to CMEX summarising the important risk factors and a systematic approach to management. Whilst this review has been all encompassing, there are some limitations. Heterogeneity of the studies included in the paper have made performing a meta-analysis tricky and often difficult to compare data across different types of studies. Most of the studies are retrospective and with rates of CMEX being generally low, there are inadequately statically powered studies. There have been technological changes in CT and MRI, especially with use of non-ionic CM, increased understanding of the risk factors and use of EDAs as well as systems which halt injection if problems are encountered during infusion, all of which inform update on the previous guidelines. However, there remain important areas where further research would be merited. There is no data available on patient experience

Table 3 Risk factors

\begin{tabular}{ll}
\hline Technique & Patient \\
\hline - Less optimal injection sites including lower limb and small distal veins & $\bullet$ Inability of patient to communicate \\
- Large volume of contrast medium & $\bullet$ Fragile or damaged veins \\
- High osmolarity contrast media & $\bullet$ Compromised lymphatic and/or venous drainage \\
- Viscous contrast media & $\bullet$ Obesity \\
\hline
\end{tabular}

Table 4 Preventative and minimisation measures

\begin{tabular}{|c|c|}
\hline & $\begin{array}{l}\text { Grade of } \\
\text { recommendation* }\end{array}$ \\
\hline Meticulous cannula insertion technique using an appropriate size upper arm vein is preferred & $\mathrm{C}$ \\
\hline An appropriately sized cannula for the vein and anticipated flow rate & B \\
\hline Test injection with saline prior to contrast administration & $\mathrm{D}$ \\
\hline Warming of the contrast medium, especially for higher viscosity compounds & B \\
\hline Minimising the volume of contrast administered based upon the indication and patient size & B \\
\hline Use of correct flow rates and pressures appropriate to the specific catheter, especially when using central venous catheters & $\mathrm{B}$ \\
\hline $\begin{array}{l}\text { Effective detection protocol which allows early diagnosis, this ranges from direct observation to considering use of extrava- } \\
\text { sation detection accessories in high-risk patients }\end{array}$ & B \\
\hline
\end{tabular}

*Grade of recommendation (see Appendix 2) 
Table 5 Treatment of contrast media extravasation (CMEX)

Mechanisms and discussion of evidence

\section{Conservative Treatments}

Aspiration of contrast whilst cannula still in place prior to removal.

Raise the affected limb if possible.

Cooling of the region.A cold compress 15 to 60 minutes three times per day for a period of 3 to 4 days [1].

Warming of the region.

Heparin ointment dressing with cooling (where the dermis is intact).

Topical non-steroidal anti-inflammatory drugs (NSAIDs).

\section{Invasive treatments}

Hyaluronic acid injection (HYLA). Dose of between 5-250 Units is thought to be most effective [15].

Aspiration \& irrigation: essentially "wash-out" using stab incisions around the area of concern under local anaesthetic and extravasate aspirated with blunt suction cannulas. This is followed by irrigation (performed within 6 hours).

Manual squeezing technique: manual expression of extravasate after various punctures/stab incisions (e.g. 5-10 stabs with $18 \mathrm{G}$ needle).

Fasciotomy and compartment release
This reduces the volume of contrast extravasate and reduce pressure [57].

Minimise oedema by reducing hydrostatic pressure and promoting drainage [58].

Anti-inflammatory effect via vasoconstriction and is widely recommended when treating CMEX [1, 13, 59].

Controversially, some think that cooling can delay resorption of extravasate, and warming can lead to vasodilation hence increasing contrast media (CM) resorption. Hastings-Tolsma et al. conducted studies with saline, assessing extravasation by the effect of both warming and cooling extremities [60]. No significant difference was observed between groups in terms of surface area induration, or evidence of extravasate taking longer to resorb when warm solution was applied.

Anecdotal use has been suggested in a recent review paper by Mandlik et al. [12].

Evidence only pertains to the analgesic effects on acute pain and not specifically extravasation [61].

This mucopolysaccharide is injected directly into the site of CMEX and is thought to work by enzymatically cleaving structures of the interstitium thus promoting resorption into vessels and lymphatics. Limited evidence supporting its use [62]. Indeed, some data does not support its use as some animal models have shown an increased inflammatory response [63]. Overall, not considered routine treatment (only off-label use e.g. inoperable patients with compartment syndrome due to CMEX).

There is variation as to the exact technique, based on a retrospective study by Gault in 96 patients with extravasation, 44 were successfully treated [64]. However, only 1 patient had a CMEX (others being chemotherapy agents etc.) meaning it may be less applicable to CMEX. Further case series of 11 patients by Vandeweyer et al. described successful use, although this was with high osmolarity, ionic agents [65]. Overall, this is a mechanistically plausible method but without strong evidence base for routine use.

Study by Tsai et al. of 8 cases who developed vascular compromise with $50-80 \mathrm{ml}$ of non-ionic, low osmolarity extravasate demonstrated satisfactory healing using this method [66]. A similar study by Kim et al. with 23 cases (no control group) of extravasate $>50 \mathrm{ml}$ also showed satisfactory response after 1 week follow-up although there was immediate temporary mild blistering [67]. A similar technique whereby multiple stab incisions were made for large volume extravasations was found to be successful in a case report by Raveendran et al. [68] Similar to other techniques, limited data available to support use of this, but the simplicity is attractive and more comparative data would help assess efficacy.

Considered the definitive surgical treatment when a CMEX is complicated by neurovascular compromise or compartment syndrome. A retrospective study by Fallscheer et al., identified seven patients required fasciotomy [54]. Delay to refer to plastic surgery by $>300$ minutes is the greatest risk factor contributing to complications postoperatively. of CMEX and this is an important impact to explore as the patient may refuse to have CM in the future. Long-term follow-up of patients after CMEX and prospective trials with CMEX interventions are also not well-researched.
Important questions to ask are appropriate time interval when to re-scan after a CMEX, impact on workflow and cost implications. 
Table 6 Suggested protocol for management of contrast media extravasation

\section{Grade of recommendation *}

Conservative

- Stop injection and scan-classify as mild, moderate, or severe

- Accurate documentation, demarcate area affected and consult responsible physician

- Mild cases: limb elevation, ice packs, monitor patient 2-4 hourly. If improving, then discharge. If no improvement, then requires surgical opinion

- Radiographic documentation for moderate and severe cases - two orthogonal views or cross-sectional imaging can help assess compartmentalization and extent of extravasation

- Record extravasation as a complication in radiology C report and the local incident reporting system

- Patient information leaflet should be given to patient

- Follow-up appointment, if necessary

$\mathrm{D}$

C

$\mathrm{C}$

Active

- If severe injury (e.g. neurovascular compromise, compartment syndrome, tissue necrosis) suspected then urgently seek advice of a surgeon

- Surgical opinion also recommended for extravasate $>150 \mathrm{ml}$

*Grade of recommendation (see Appendix 2)

Supplementary Information The online version contains supplementary material available at https://doi.org/10.1007/s00330-021-08433-4.

Funding The authors state that this work has not received any funding.

\section{Declarations}

Guarantor The scientific guarantor of this publication is Olivier Clément.

Conflict of interest The authors of this manuscript declare relationships with the following companies:

Giles Roditi: none.

Nadir Khan: none.

Aart J. van der Molen: Bayer Healthcare.

Marie-France Bellin: none.

Michele Bertolotto: none.

Torkel Brismar: none.

Jean-Michel Correas: Bracco, Guerbet.

Ilona A. Dekkers: none.

Remy W.F. Geenen: none.

Gertraud Heinz-Peer: none.

Andreas H. Mahnken: none.

Carlo C. Quattrocchi: none.

Alexander Radbruch: Bracco, Guerbet, Bayer, GE.

Peter Reimer: none.

Laura Romanini: none.

Fulvio Stacul: none.

Henrik S. Thomsen: pending.

Olivier Clément: Bracco, Guerbet, Bayer Healthcare.
M.F.B., T.B. and F.S. are members of the European Radiology Editorial Board. They have not taken part in the review or selection process of this article.

Statistics and biometry One of the authors has significant statistical expertise (Ilona Dekkers).

Informed consent Not applicable.

Ethical approval Not applicable.

Study subjects or cohorts overlap Not applicable.

Methodology

- retrospective

Open Access This article is licensed under a Creative Commons Attribution 4.0 International License, which permits use, sharing, adaptation, distribution and reproduction in any medium or format, as long as you give appropriate credit to the original author(s) and the source, provide a link to the Creative Commons licence, and indicate if changes were made. The images or other third party material in this article are included in the article's Creative Commons licence, unless indicated otherwise in a credit line to the material. If material is not included in the article's Creative Commons licence and your intended use is not permitted by statutory regulation or exceeds the permitted use, you will need to obtain permission directly from the copyright holder. To view a copy of this licence, visit http://creativecommons.org/licenses/by/4.0/.

\section{References}

1. Bellin M-F, Jakobsen JA, Tomassin I et al (2002) Contrast medium extravasation injury: guidelines for prevention and management. Eur Radiol 12:2807-2812

2. Hwang EJ, Shin C-I, Choi YH, Park CM (2018) Frequency, outcome, and risk factors of contrast media extravasation in 142,651 intravenous contrast-enhanced CT scans. Eur Radiol 28:5368-5375

3. Heshmatzadeh Behzadi A, Farooq Z, Newhouse JH, Prince MR (2018) MRI and CT contrast media extravasation. Medicine (Baltimore) 97.https://doi.org/10.1097/MD.0000000000010055

4. Wilson BG (2011) Contrast media-induced compartment syndrome. Radiol Technol 83:63-77

5. Benson LS, Sathy MJ, Port RB (1996) Forearm compartment syndrome due to automated injection of computed tomography contrast material. J Orthop Trauma 10:433-436

6. Belzunegui T, Louis CJ, Torrededia L, Oteiza J (2011) Extravasation of radiographic contrast material and compartment syndrome in the hand: a case report. Scand J Trauma Resusc Emerg Med 19:9

7. Alami Z, Nasri S, Ahid S, Kacem HH (2015) Extravasation of contrast medium during CT examination: an observational casecontrol study. Pan Afr Med J 20. https://doi.org/10.11604/pamj. 2015.20.89.3276

8. Kyung E-J, Ryu J-H, Kim E-Y (2013) Evaluation of adverse reactions to contrast media in the hospital. Br J Radiol 86.https://doi. org/10.1259/bjr.20130418

9. van der Molen AJ, Reimer P, Dekkers IA et al (2018) Post-contrast acute kidney injury - Part 1: definition, clinical features, incidence, role of contrast medium and risk factors : Recommendations for updated ESUR Contrast Medium Safety Committee guidelines. Eur Radiol 28:2845-2855 
10. van der Molen AJ, Reimer P, Dekkers IA et al (2018) Post-contrast acute kidney injury. Part 2: risk stratification, role of hydration and other prophylactic measures, patients taking metformin and chronic dialysis patients. Eur Radiol 28:2856-2869

11. Ding S, Meystre NR, Campeanu C, Gullo G (2018) Contrast media extravasations in patients undergoing computerized tomography scanning: a systematic review and meta-analysis of risk factors and interventions. JBI Database System Rev Implement Rep 16:87-116

12. Mandlik V, Prantl L, Schreyer AG (2019) Contrast media extravasation in CT and MRI - a literature review and strategies for therapy. Rofo 191:25-32

13. Hrycyk J, Heverhagen JT (1987) Boehm I (2019) What you should know about prophylaxis and treatment of radiographic and magnetic resonance contrast medium extravasation. Acta Radiol 60:496-500

14. Guyatt GH, Oxman AD, Kunz R et al (2011) GRADE guidelines: 2. Framing the question and deciding on important outcomes. J Clin Epidemiol 64:395-400

15. Dijkmans PA, Visser CA, Kamp O (2005) Adverse reactions to ultrasound contrast agents: is the risk worth the benefit? Eur J Echocardiogr 6:363-366

16. Rosado E, Riccabona M (2016) Off-label use of ultrasound contrast agents for intravenous applications in children: analysis of the existing literature. J Ultrasound Med 35:487-496

17. Hu C, Feng Y, Huang P, Jin J (2019) Adverse reactions after the use of SonoVue contrast agent. Medicine (Baltimore) 98.https:// doi.org/10.1097/MD.0000000000017745

18. Lewis GB, Hecker JF (1991) Radiological examination of failure of intravenous infusions. Br J Surg 78:500-501

19. Sakellariou S, Li W, Paul MC, Roditi G (2016) Rôle of contrast media viscosity in altering vessel wall shear stress and relation to the risk of contrast extravasations. Med Eng Phys 38:1426-1433

20. Selek H, Ozer H, Aygencel G, Turanli S (2007) Compartment syndrome in the hand due to extravasation of contrast material. Arch Orthop Trauma Surg 127:425-427

21. Tonolini M, Campari A, Bianco R (2012) Extravasation of radiographic contrast media: prevention, diagnosis, and treatment. Curr Probl Diagn Radiol 41:52-55

22. Cleary N, McNulty JP, Foley SJ, Kelly E (2017) An investigation into current protocols and radiographer opinions on contrast extravasation in Irish CT departments. Radiography (Lond) 1995 23:e87-e92

23. Dykes TM, Bhargavan-Chatfield M, Dyer RB (2015) Intravenous contrast extravasation during CT: a national data registry and practice quality improvement initiative. J Am Coll Radiol 12:183-191

24. Birnbaum BA, Nelson RC, Chezmar JL, Glick SN (1999) Extravasation detection accessory: clinical evaluation in 500 patients. Radiology 212:431-438

25. Nelson RC, Anderson FA, Birnbaum BA et al (1998) Contrast media extravasation during dynamic CT: detection with an extravasation detection accessory. Radiology 209:837-843

26. Powell CC, Li JM, Rodino L, Anderson FA (2000) A new device to limit extravasation during contrast-enhanced CT. AJR Am J Roentgenol 174:315-318

27. Saade C, Brennan P (2011) Clinical implementation of the new MEDRAD XDS contrast extravasation detector for multidetector computed tomography. J Med Imaging Radiat Sci 42:179-182

28. Hoff L, Brabrand K, Andersen NB, Medhus S (2008) Monitoring $\mathrm{X}$-ray contrast agent injections with Doppler ultrasound. IEEE Ultrason Symp 2008:13-16

29. Teo M, Ong C, Ying A, Hng M (2015) Extravasation of contrast medium during CT scanning - tracking and reduction of rate of extravasation. https://posterng.netkey.at/esr/viewing/ index.php? module $=$ viewing_poster \&task $=$ viewsection \& $\mathrm{pi}=$ $126853 \& \mathrm{ti}=422983 \& \mathrm{si}=1478 \&$ searchkey $=$. Accessed 16 Oct 2019

30. Cohan RH (1995) Considering large-volume intravenous injections of iodinated contrast media for contrast-enhanced CT (head and body). AJR Am J Roentgenol 164:1292

31. Tonolini M (2016) Contrast medium extravasation: the importance of radiographic assessment. Curr Probl Diagn Radiol 45:236-237

32. Johnson PT, Christensen GM, Fishman EK (2014) I.v. contrast administration with dual source 128-MDCT: a randomized controlled study comparing 18 -gauge nonfenestrated and 20-gauge fenestrated catheters for catheter placement success, infusion rate, image quality, and complications. AJR Am J Roentgenol 202:1166-1170

33. Wienbeck S, Fischbach R, Kloska SP et al (2010) Prospective study of access site complications of automated contrast injection with peripheral venous access in MDCT. AJR Am J Roentgenol 195:825-829

34. Sinan T, Al-Khawari H, Chishti FA, Al Saeed OM, Sheikh M (2005) Contrast media extravasation: manual versus power injector. Med Princ Pract 14:107-110

35. Schwab SA, Uder M, Anders K et al (2009) Peripheral intravenous power injection of iodinated contrast media through $22 \mathrm{G}$ and $20 \mathrm{G}$ cannulas: can high flow rates be achieved safely? A clinical feasibility study. Rofo 181:355-361

36. Amaral JG, Traubici J, BenDavid G et al (2006) Safety of power injector use in children as measured by incidence of extravasation. AJR Am J Roentgenol 187:580-583

37. Plumb AAO, Murphy G (2011) The use of central venous catheters for intravenous contrast injection for CT examinations. Br J Radiol 84:197-203

38. Buijs SB, Barentsz MW, Smits MLJ et al (2017) Systematic review of the safety and efficacy of contrast injection via venous catheters for contrast-enhanced computed tomography. Eur J Radiol Open 4:118-122

39. Radiological Society of the Netherlands (2019) Guideline safe use of contrast media part 2. Radiological Society of the Netherlands, Utrecht. https://www.radiologen.nl/sites/default/files/secti es/abdominale/richtlijnen/guideline_safe_use_of_contrast_media_ part_2.pdf. Accessed 26 Oct 2020

40. Alexander MD, Morrison HL (2012) Power-injectable ports: safety during placement, therapeutic use, and contrast administration during computed tomography procedures. J Vasc Access 13:432-437

41. Goltz JP, Machann W, Noack C et al (2011) Feasibility of power contrast injections and bolus triggering during CT scans in oncologic patients with totally implantable venous access ports of the forearm. Acta Radiol 52:41-47

42. Rigsby CK, Gasber E, Seshadri R et al (2007) Safety and efficacy of pressure-limited power injection of iodinated contrast medium through central lines in children. AJR Am J Roentgenol 188:726-732

43. Barrera CA, White AM, Shepherd AM et al (2019) Contrast Extravasation using power injectors for contrast-enhanced computed tomography in children: frequency and injury severity. Acad Radiol. https://doi.org/10.1016/j.acra.2019.04.008

44. Kok M, Mihl C, Hendriks BMF et al (2016) Patient comfort during contrast media injection in coronary computed tomographic angiography using varying contrast media concentrations and flow rates: results from the EICAR trial. Invest Radiol $51: 810-815$

45. Shaqdan K, Aran S, Thrall J, Abujudeh H (2014) Incidence of contrast medium extravasation for CT and MRI in a large academic medical centre: a report on 502,391 injections. Clin Radiol 69:1264-1272 
46. Moreno CC, Pinho D, Nelson RC et al (2013) Lessons learned from 118,970 multidetector computed tomographic intravenous contrast material administrations: impact of catheter dwell time and gauge, catheter location, rate of contrast material administration, and patient age and sex on volume of extravasate. J Comput Assist Tomogr 37:286-288

47. Rupp JD, Ferre RM, Boyd JS et al (2016) Extravasation risk using ultrasound-guided peripheral intravenous catheters for computed tomography contrast administration. Acad Emerg Med 23:918-921

48. Cohan RH, Leder RA, Herzberg AJ et al (1991) Extravascular toxicity of two magnetic resonance contrast agents. Preliminary experience in the rat. Invest Radiol 26:224-226

49. Cohan RH, Ellis JH, Garner WL (1996) Extravasation of radiographic contrast material: recognition, prevention, and treatment. Radiology 200:593-604

50. Miles SG, Rasmussen JF, Litwiller T, Osik A (1990) Safe use of an intravenous power injector for CT: experience and protocol. Radiology 176:69-70

51. Davenport MS, Wang CL, Bashir MR et al (2012) Rate of contrast material extravasations and allergic-like reactions: effect of extrinsic warming of low-osmolality iodinated CT contrast material to $37^{\circ} \mathrm{C}$. Radiology $262: 475-484$

52. Stroeder J, Fries P, Raczeck P et al (2020) Prospective safety evaluation of automated iomeprol 400 injections for CT through peripheral venous cannulas. Clin Radiol 75:396.e1-396.e6

53. van Veelen NM, Link B-C, Donner G et al (2020) Compartment syndrome of the forearm caused by contrast medium extravasation: a case report and review of the literature. Clin Imaging 61:58-61

54. Fallscheer P, Kammer E, Roeren T, Meuli-Simmen C (2007) Injury to the upper extremity caused by extravasation of contrast medium: a true emergency. Scand J Plast Reconstr Surg Hand Surg 41:26-32

55. Sbitany H, Koltz PF, Mays C et al (2010) CT contrast extravasation in the upper extremity: strategies for management. Int $\mathrm{J}$ Surg Lond Engl 8:384-386

56. Wang CL, Cohan RH, Ellis JH et al (2007) Frequency, management, and outcome of extravasation of nonionic iodinated contrast medium in 69,657 intravenous injections. Radiology 243:80-87

57. Khan MS, Holmes JD (2002) Reducing the morbidity from extravasation injuries. Ann Plast Surg 48:628-632; discussion 632. https://doi.org/10.1097/00000637-200206000-00011
58. Nicola R, Shaqdan KW, Aran S et al (2016) Contrast media extravasation of computed tomography and magnetic resonance imaging: management guidelines for the radiologist. Curr Probl Diagn Radiol 45:161-164

59. Beckett KR, Moriarity AK, Langer JM (2015) Safe use of contrast media: what the radiologist needs to know. Radiogr Rev Publ Radiol Soc N Am Inc 35:1738-1750

60. Hastings-Tolsma MT, Yucha CB, Tompkins J et al (1993) Effect of warm and cold applications on the resolution of i.v. infiltrations. Res Nurs Health 16:171-178

61. Massey T, Derry S, Moore RA, McQuay HJ (2010) Topical NSAIDs for acute pain in adults. Cochrane Database Syst Rev CD007402. https://doi.org/10.1002/14651858.CD007402.pub2

62. Federle MP, Chang PJ, Confer S, Ozgun B (1998) Frequency and effects of extravasation of ionic and nonionic CT contrast media during rapid bolus injection. Radiology 206:637-640

63. McAlister WH, Kissane JM (1990) Comparison of soft tissue effects of conventional ionic, low osmolar ionic and nonionic iodine containing contrast material in experimental animals. Pediatr Radiol 20:170-174

64. Gault DT (1993) Extravasation injuries. Br J Plast Surg 46:91-96

65. Vandeweyer E, Heymans O, Deraemaecker R (2000) Extravasation injuries and emergency suction as treatment. Plast Reconstr Surg 105:109-110

66. Tsai YS, Cheng SM, Ng SP et al (2007) Squeeze maneuver: an easy way to manage radiological contrast-medium extravasation. Acta Radiol 48:605-607

67. Kim SM, Cook KH, Lee IJ et al (2017) Computed tomography contrast media extravasation: treatment algorithm and immediate treatment by squeezing with multiple slit incisions. Int Wound $\mathrm{J}$ 14:430-434

68. Raveendran S, Rajendra Benny K, Monica S et al (2019) Multiple Stab incisions and evacuation technique for contrast extravasation of the hand and forearm. J Hand Surg 44:71.e1-71.e5

Publisher's note Springer Nature remains neutral with regard to jurisdictional claims in published maps and institutional affiliations. 